九州大学学術情報リポジトリ

Kyushu University Institutional Repository

\title{
CATEGORICAL RELATIONAL DATABASE MODELS
}

Kawahara, Yasuo

Department of Mathematics, Kyushu University

https://doi.org/10.5109/13360

出版情報: Bulletin of informatics and cybernetics. 21 (1/2), pp.93-99, 1984-03. Research Association of Statistical Sciences

バージョン :

権利関係 : 


\title{
CATEGORICAL RELATIONAL DATABASE MODELS
}

\section{By}

\author{
Yasuo KaWAHARA*
}

\begin{abstract}
A category of relational models and its quotient category are defined and their basic properties are discussed. Moreover a categorical join dependency and a category of adjoint models, which dualize the notion of relational models, are studied.
\end{abstract}

\section{Introduction}

Theory of categories $[1,8]$ founded by S. Mac Lane and S. Eilenberg can be applied to various fields of mathematics and has the advantage that it serves a global point of view for logical structures of many mathematical objects. For instance category theory has. been a useful tool in studying theories of automata, mathematical languages, systems, graphs and programmings $[1,2,3,4,5]$ as mathematical foundations of computer science.

The purpose of this paper is to investigate a categorical aspect of relational detabase models. This categorical viewpoint for theory of detabase models was initiated by the work of A. Kato $[6,7]$. Given an image factorization system $(\mathcal{E}, \mathscr{M})$ of a category $\mathcal{C}$ and a functor $X: \mathfrak{X} \rightarrow \mathcal{C}$, we define relational models and their morphisms in section 2 . Then we can naturally obtain a category $R(X)$ of relational models and morphisms between them. Also we show some basic properties of the category $R(X)$ of relational models and consider an equivalence relation on the class of all morphisms in $R(X)$ to obtain a quotient category $R[X]$ of $R(X)$. In section 3 a join dependency associated with a family of natural transformations $k_{\alpha}: X \rightarrow X_{\alpha}(\alpha \in \Lambda)$ is introduced. The categorical join dependency is a reasonable abstraction of the usual join dependency and it induces a left adjoint functor from $R(X)$ into a full subcategory $D(X)$ of $R(X)$ consisting of all join dependent models. As in Kato [6] it turns out that the join dependency of a relational model is regarded as a notion in the quotient category $R[X]$. In the final section we introduce a notion of adjoint models as a dual of relational models and their morphisms in case that a functor $X: \mathscr{X} \rightarrow \mathcal{C}$ has a right adjoint functor $Y: \mathcal{C} \rightarrow \mathscr{C}$ and $\mathscr{X}$ has an image factorization system $\left(\mathcal{E}^{*}, \mathscr{M}^{*}\right)$. Then the category $R\{Y\}$ of adjoint models is naturally constructed and we extend Kato's result [6] that $R\{Y\}$ is equivalent to $R[X]$ as categories.

\section{Categories of Relational Models}

Relational database models due to E.F. Codd are defined as subsets of a cartesian product of sets indexed by a set of atributes. In this section we will generalize the

\footnotetext{
* Department of Mathematics, Kyushu University 33, Fukuoka 812, Japan.
} 
notion of relational database models and define a category consisting of them. Throughout the rest of the paper we assume that an image factorization system $(\mathcal{E}, \mathscr{M})$ in a category $\mathcal{C}$ and a functor $X: \mathscr{X} \rightarrow \mathcal{C}$ are given.

Definition 2.1. A (relational) model (with respect to $X: X \rightarrow \mathcal{C}$ ) is defined to be a pair $(Q, q)$ of an object $Q$ in $\mathscr{X}$ and a monomorphism $q: \bar{Q} \rightarrow X Q$ in $\mathcal{C}$ such that $q \in \mathscr{M}$. A morphism $f:(Q, q) \rightarrow(R, r)$ of a model $(Q, q)$ into another model $(R, r)$ is defined to be a morphism $f: Q \rightarrow R$ in $\mathscr{X}$ such that there exists a morphism $\bar{f}: \bar{Q} \rightarrow \bar{R}$ in $\mathcal{C}$ rendering the following square commutative :

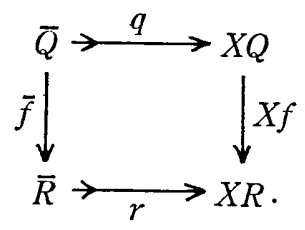

(The uniqueness of $\bar{f}: \bar{Q} \rightarrow \bar{R}$ follows from the injectivity of $r: \bar{R} \rightarrow X R$. An arrow “ $\rightarrow$ " represents a monomorphism in $\mathscr{M}$.)

Let $R(X)$ be the category of all models and all morphisms between them. Identity morphisms and the composition of morphisms in $R(X)$ are given in a trivial fashion.

LEMMA 2.2. A morphism $f:(Q, q) \rightarrow(R, r)$ is an isomorphism in $R(X)$ if and only if $f: Q \rightarrow R$ is an isomorphism in $\mathscr{X}$ and $\bar{f}: \bar{Q} \rightarrow \bar{R}$ is an epimorphism in $\mathcal{E}$.

By analogy with A. Kato [6] we define an equivalence relation on the class of all morphisms in category $R(X)$ as follows:

DeFinition 2.3. Let $f_{1}, f_{2}:(Q, q) \rightarrow(R, r)$ be two morphisms in $R(X)$. We say that $f_{1}$ is equivalent to $f_{2}$, denoted by $f_{1} \sim f_{2}:(Q, q) \rightarrow(R, r)$, if $\bar{f}_{1}: \bar{Q} \rightarrow \bar{R}$ and $\bar{f}_{2}: \bar{Q} \rightarrow \bar{R}$ (in Definition 2.1) are identical, that is, $\bar{f}_{1}=\bar{f}_{2}$.

It is obvious that the relation " $\sim$ " is an equivalence relation. The next proposition states that this equivalence relation " $\sim$ " is preserved by the composition of morphisms in $R(X)$.

Proposition 2.4. If $f_{1} \sim f_{2}:(Q, q) \rightarrow(R, r)$ and $g_{1} \sim g_{2}:(R, r) \rightarrow(S, s)$ in $R(X)$ then $g_{1} f_{1} \sim g_{2} f_{2}:(Q, q) \rightarrow(S, s)$.

We are now ready to obtain a quotient category $[8 ;$ p. 51$]$ of $R(X)$ classifying morphisms by the equivalence relation " $\sim$ " defined above. We denote the quotient category by $R[X]$. That is, objects in $R[X]$ are the same ones as in $R(X)$ and a morphism $[f]:(Q, q) \rightarrow(R, r)$ in $R[X]$ is an equivalence class of a morphism $f:(Q, q) \rightarrow$ $(R, r)$ in $R(X)$.

Proposition 2.5. Let $f:(Q, q) \rightarrow(R, r)$ be a morphism in $R(X)$. If $f: Q \rightarrow R$ is an isomorphism in $X$ and if there exists a morphism $g:(R, r) \rightarrow(Q, q)$ with $f g \sim 1_{R}$, then $f:(Q, q) \rightarrow(R, r)$ is an isomorphism in $R(X)$.

In the above proposition the existence of a morphism $g:(R, r) \rightarrow(Q, q)$ with $f g \sim 1_{R}$ : $(R, r) \rightarrow(R, r)$ means that $[f]:(Q, q) \rightarrow(R, r)$ is a retraction $[5 ;$ p. 19] in $R[X]$.

Corollary 2.6. Let $f:(Q, q) \rightarrow(R, r)$ be a morphism in $R(X)$. If $f: Q \rightarrow R$ is an isomorphism in $\mathcal{X}$ and if $[f]:(Q, q) \rightarrow(R, r)$ is an isomorphism in $R[X]$, then $f:(Q, q)$ $\rightarrow(R, r)$ is an isomorphism in $R(X)$.

By using the diagonal fill-in lemma $[1$, p. 39] we have the following lemma. 
LEMMA 2.7. Let $f:(Q, q) \rightarrow(R, r)$ be a morphism in $R(X)$. If there exists a morphism $g: R \rightarrow Q$ with $g f=1_{Q}$ and if $\bar{f}: \bar{Q} \rightarrow \bar{R}$ is in $\mathcal{E}$, then $[f]:(Q, q) \rightarrow(R, r)$ is an isomorphism in $R[X]$.

EXAMPLE 2.8. Let $\mathcal{C}$ be a category with products and $A$ a set. $A$ is regarded as a discrete category. Denote by $\mathcal{C}^{A}$ the functor category of all functors from $A$ into $\mathcal{C}$, i. e., $\mathcal{C}^{A}$ is a product cotegory of $A$ copies of $\mathcal{C}$. Let $\pi_{A}: \mathcal{C}^{A} \rightarrow \mathcal{C}$ be the product functor $\left(\left\langle S_{a}\right\rangle_{a \in A^{\mapsto}} \rightarrow \pi_{a} S_{a}\right)$. Then the category $R\left(\pi_{A}\right)$ of relational models is obtained and the case $\mathcal{C}=$ Set was considered by Kato $[6]$.

\section{Generalized Join Dependencies}

In [6] Kato described that observations of join dependencies about relational models as stated in Example 2.8 are sufficient if they are considered in quotient category $R[X]$. This section generalizes the result due to Kato [6] for the category of relational models defined in the previous section.

As in the section 2 , we assume that an image factorization $\operatorname{system}(\mathcal{E}, \mathscr{M})$ in a category $\mathcal{C}$ and a functor $X: \mathfrak{X} \rightarrow \mathcal{C}$ are given. Moreover supposed that category $\mathcal{C}$ has products and pullbacks, and supposed that a family of functors $X_{\alpha}: \mathscr{X} \rightarrow \mathcal{C}(\alpha \in \Lambda)$ and a family of natural transformations $k_{\alpha}: X \rightarrow X_{\alpha}(\alpha \in \Lambda)$ are given.

Let $(Q, q)$ be an object of category $R(X)$. Then consider an $(\mathcal{E}, \mathscr{M})$-factorization of the composite $k_{\alpha} q: \bar{Q} \rightarrow X Q \rightarrow X_{\alpha} Q(\alpha \in \Lambda)$, which is rendered by the commutative square

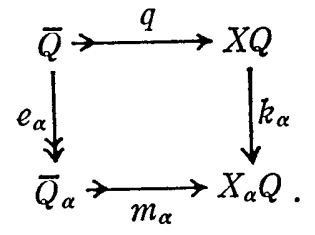

(Notice that $k_{\alpha}=k_{\alpha, Q}: X Q \rightarrow X_{\alpha} Q$. An arrow “ $\rightarrow$ " represents an epimorphism in $\mathcal{E}$.) Let $p r_{\alpha}: \pi_{\alpha} X_{\alpha} Q \rightarrow X_{\alpha} Q(\alpha \in \Lambda)$ and $\overline{p r}_{\alpha}: \pi_{\alpha} \bar{Q}_{\alpha} \rightarrow \bar{Q}_{\alpha}(\alpha \in \Lambda)$ be systems of projections of products $\pi_{\alpha} X_{\alpha} Q$ and $\pi_{\alpha} \bar{Q}_{\alpha}$, respectiqely. We denote by $\mathrm{T}_{\alpha} k_{\alpha}: X Q \rightarrow \pi_{\alpha} X_{\alpha} Q$ a unique morphism with $p r_{\alpha}\left(T_{\alpha} k_{\alpha}\right)=k_{\alpha}$ for each $\alpha \in \Lambda$ and by $\pi_{\alpha} m_{\alpha}: \Pi_{\alpha} \bar{Q}_{\alpha} \rightarrow \pi_{\alpha} X_{\alpha} Q$ a unique morphism with $p r_{\alpha}\left(\pi_{\alpha} m_{\alpha}\right)=m_{\alpha} \overline{p r}_{\alpha}$ for each $\alpha \in \Lambda$. Construct a pullback of two morphisms $\mathrm{T}_{\alpha} k_{\alpha}$ and $\pi_{\alpha} m_{\alpha}$ with common codomain.

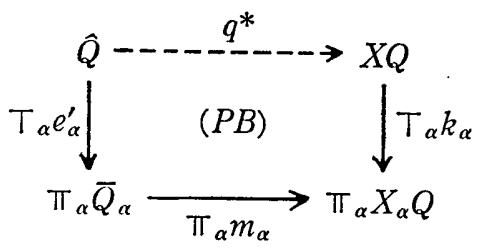

Trivially $q^{*}$ is in $\mathscr{M}[1$, Exercise $2.4 .11 ; 2$, Proposition 2.11$]$ since $m_{\alpha}$ is in $\mathscr{M}$ for each $\alpha \in \Lambda$. Hence we have a new object $\left(Q, q^{*}\right)$ in $R(X)$. By the universal property of pullbacks there exists a unique morphism $\bar{\eta}: \bar{Q} \rightarrow \hat{Q}$ in $\mathscr{X}$ making the following daigram commute. 


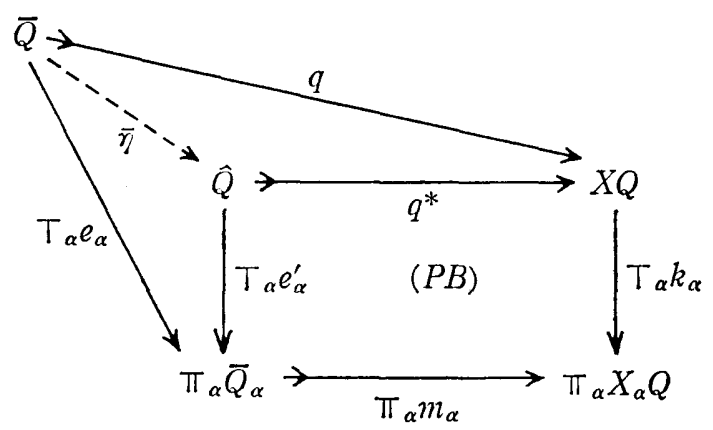

This implies that $1_{Q}:(Q, q) \rightarrow\left(Q, q^{*}\right)$ is a morphism in $R(X)$. Note that $1_{Q}: Q \rightarrow Q$ is an identity morphism in $\mathscr{X}$ but $1_{Q}:(Q, q) \rightarrow\left(Q, q^{*}\right)$ is not always an identity morphism in $R(X)$. It follows at once from the construction of $q^{*}$ that $q^{* *}=q^{*}$ since $e_{\alpha}^{\prime}$ is in $\mathcal{E}$ for each $\alpha \in \Lambda$.

Proposition 3.1. Let $(Q, q)$ and $(R, r)$ be two objects in $R(X)$. If $f:(Q, q) \rightarrow\left(R, r^{*}\right)$ is a morphism in $R(X)$, then so is $f:\left(Q, q^{*}\right) \rightarrow\left(R, r^{*}\right)$.

CoRollary 3.2. If $f:(Q, q) \rightarrow(R, r)$ is a morphism in $R(X)$, then so is $f:\left(Q, q^{*}\right)$ $\rightarrow\left(R, r^{*}\right)$.

The last corollary shows that $*$ gives a functor called the join functor:

$$
\begin{aligned}
& \text { * }: R(X) \longrightarrow R(X) \\
& (Q, q) \quad\left(Q, q^{*}\right) \\
& f \downarrow \longmapsto \downarrow f \\
& (R, r) \quad\left(R, r^{*}\right) .
\end{aligned}
$$

During the rest of this section we assume that $\top_{\alpha} k_{\alpha}: X Q \rightarrow \pi_{\alpha} X_{\alpha} Q$ is a monomorphism for each object $Q$ in $\mathscr{X}$. The following results are basic properties of the join functor $*: R(X) \rightarrow R(X)$.

LEMMA 3.3. Let $f, g:(Q, q) \rightarrow(R, r)$ be two morphisms in $R(X)$. If $f \sim g:(Q, q) \rightarrow$ $(R, r)$, then $f \sim g:\left(Q, q^{*}\right) \rightarrow\left(R, r^{*}\right)$.

Corollary 3.4. An object $(Q, q)$ in $R(X)$ is join dependent $(J D)$, that is, $q=q^{*}$ as a subobject of $X Q$ if and only if $\left[1_{Q}\right]:(Q, q) \rightarrow\left(Q, q^{*}\right)$ is an isomorphism in $R[X]$.

THEOREM 3.5. Let $(Q, q)$ and $(R, r)$ be two object in $R(X)$. If $(R, r)$ is isomorphic to $(Q, q)$ in $R[X]$ and if $q=q^{*}(J D)$, then $r=r^{*}(J D)$.

Let $D(X)$ be the full subcategory of $R(X)$ consisting of all objects $(Q, q)$ with $q=q^{*}$ $(J D)$. Then Proposition 3.1 indicates that the inclusion functor $I: D(X) \subset R(X)$ is a right adjoint of the join functor $*: R(X) \rightarrow D(X)$ :

$$
\frac{(Q, q) \stackrel{f}{\longrightarrow} I\left(R, r^{*}\right)}{\left(Q, q^{*}\right) \underset{f}{\longrightarrow}\left(R, r^{*}\right)} .
$$

EXAMPLE 3.6. Let $\left\{A_{\alpha} \mid \alpha \in A\right\}$ be a family of sets with $A=\bigcup_{\alpha} A_{\alpha}$. Moreover, let $\pi_{\alpha}:$ Set $^{A} \rightarrow$ Sst be a functor $\left(\left\langle S_{a}\right\rangle_{a \in A} \rightarrow \pi_{a \in A_{\alpha}} S_{a}\right)$ and $k_{\alpha}: \pi_{A} \rightarrow \pi_{\alpha}$ a natural transformation of projections $(\alpha \in \Lambda)$. Under this situation the join dependency discussed in the present section is the usual join dependency [6]. 


\section{Categories of Adjoint Models}

In this section we will define a category of adjoint models in case that a functor $X: \mathfrak{X} \rightarrow \mathcal{C}$ has a left adjoint functor $Y: \mathcal{C} \rightarrow \mathfrak{X}$. Also a sufficient condition for the category of adjoint models to be isomorphic to the quotient category $R[X]$ of relational models will be given. In addition we assume that $Y \rightarrow X: \mathcal{C} \rightarrow \mathfrak{X}$ is an adjunction $[8$, p. 78] and that $\left(\mathcal{E}^{*}, \mathscr{M}^{*}\right)$ is an image factorization system of $\mathcal{C}$.

Definition 4.1. An adjoint model (with respect to an adjunction $Y \rightarrow X: \mathcal{C} \rightarrow \mathfrak{X}$ ) is defined to be a pair $\langle\bar{Q}, \hat{q}\rangle$ of an object $\bar{Q}$ in $\mathcal{C}$ and an epimorphism $\hat{q}: Y \bar{Q} \rightarrow Q$ in $\mathscr{X}$ such that $\hat{q} \in \mathcal{E}^{*}$ and an adjoint $q: \bar{Q} \rightarrow X Q$ of $\hat{q}: Y \bar{Q} \rightarrow Q$ is in $\mathscr{M}$. A morphism $\bar{f}:\langle\bar{Q}, \hat{q}\rangle$ $\rightarrow\langle\bar{R}, \hat{r}\rangle$ of an adjoint model $\langle\bar{Q}, \hat{q}\rangle$ into another adjoint model $\langle\bar{R}, \hat{r}\rangle$ is defined to be a morphism $\bar{f}: \bar{Q} \rightarrow \bar{R}$ in $\mathcal{C}$ such that there exists a morphism $f: Q \rightarrow R$ in $\mathscr{X}$ rendering the following square commutative:

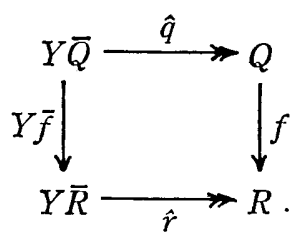

(The uniqueness of $f: Q \rightarrow R$ follows from the surjectivity of $\hat{q}: Y \bar{Q} \rightarrow Q$. Arrows " $\rightarrow$ " and " $\rightarrow$ " in $\mathscr{X}$ represent monomorphisms in $\mathscr{H}^{*}$ and epimorphisms in $\mathcal{E}^{*}$, respectively.)

Let $R\{Y\}$ be the category of all adjoint models and all morphisms between them. Identity morphisms and the composition of morphisms in $R\{Y\}$ are given as usual.

Taking an adjoint of the last commutative square we have the following commutative diagrams :

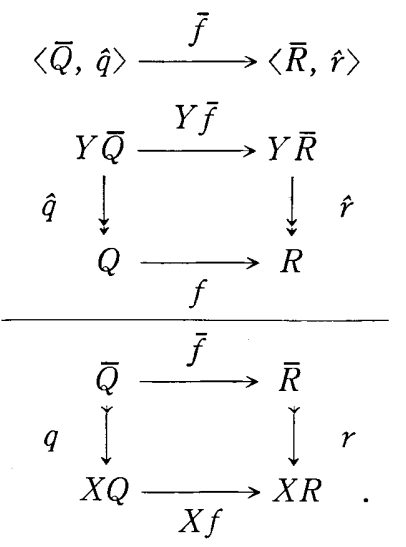

Thus we naturally obtain a functor

$$
\begin{aligned}
& F: R\{Y\} \longrightarrow R(X) \\
& \langle\bar{Q}, \hat{q}\rangle \quad(Q, q) \\
& \bar{f} \downarrow \longmapsto \downarrow f \\
& \langle\bar{R}, \hat{r}\rangle \quad(R, r) .
\end{aligned}
$$


The functor $F: R\{Y\} \rightarrow R(X)$ is called the Armstrong functor because it corresponds to a construction [9] of characteristic relations (data models) from data models defined by first order predicates satisfying equivalence laws.

Let $(R, r)$ be an object in $R(X)$ and let

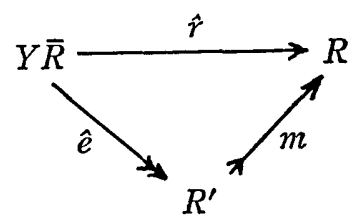

be an $\left(\mathcal{E}^{*}, \mathscr{M}^{*}\right)$-factorization of an adjoint $\hat{r}$ of $r$. Consider an adjoint of the last commutative triangle:
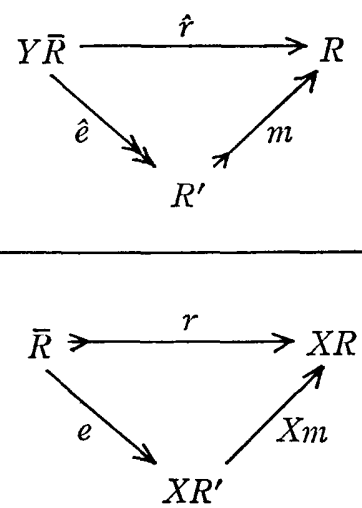

Since $X m \cdot e=r \in \mathscr{M}$ by definition of $(R, r)$ it follows that $e \in \mathscr{M}$ and hence we obtain an object $\langle\bar{R}, \hat{e}\rangle$ in $R\{Y\}$. It is trivial that $F\langle\bar{R}, \hat{e}\rangle=\left(R^{\prime}, e\right)$ and $m:\left(R^{\prime}, e\right) \rightarrow(R, r)$ is a morphism in $R(X)$, where $\bar{m}=1_{\bar{R}}$. The next lemma shows that morphism $m: F\langle\bar{R}, \hat{e}\rangle \rightarrow$ $(R, r)$ in $R(X)$ is a universal arrow [8] from $F$ to $(R, r)$ and the object $\langle\vec{R}, \hat{e}\rangle$ in $R\{Y\}$ is cofree [1] over $(R, r)$.

LEMMA 4.2. Let $\langle\bar{Q}, \hat{q}\rangle$ be an object in $R\{Y\}$ and $(R, r)$ an object in $R(X)$. For any morphism $f: F\langle\bar{Q}, \hat{q}\rangle \rightarrow(R, r)$ in $R(X)$ there exists a unique morphism $\bar{g}:\langle\bar{Q}, q\rangle \rightarrow$ $\langle\bar{R}, \hat{e}\rangle$ in $R\{Y\}$ such that the triangle
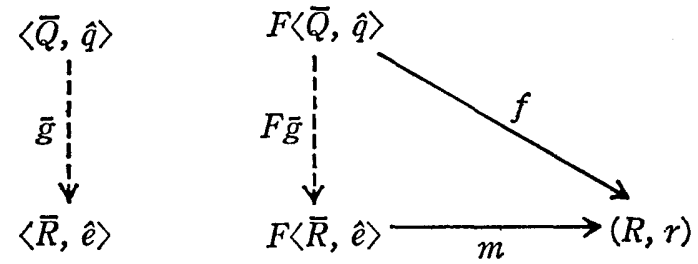

is commutative.

COROLLARY 4.3. Armstrong functor $F: R\{Y\} \rightarrow R(X)$ has a right adjoint functor $G: R(X) \rightarrow R\{Y\} \quad((R, r) \mapsto\langle\bar{R}, \hat{e}\rangle)$ and $m:\left(R^{\prime}, e\right) \rightarrow(R, r)$ is a component of count $F G \rightarrow 1_{R(X)}$ of adjunction $F \rightarrow G: R\{Y\} \rightarrow R(X)$.

Denote by $\tilde{F}: R\{Y\} \rightarrow R[X]$ the composite of Armstrong functor $F: R\{Y\} \rightarrow R(X)$ 
followed by quotient functor $P: R(X) \rightarrow R[X]$ (cf. $\S 2$ ). From definition of functor $G$ there exist a unique functor $\tilde{G}: R[X] \rightarrow R\{Y\}$ rendering the triangle of functors

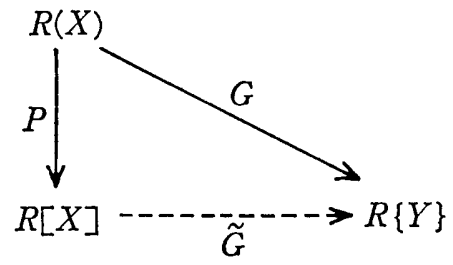

commutative. Since $G F=1_{R|X|}$ (the identity functor of $R\{Y\}$ ), it is immediate that $\tilde{G} \tilde{F}$ $=1_{R \mid Y\}}$. The totality of all morphisms $[m]: \tilde{F} \tilde{G}(R, r)=\left(R^{\prime}, e\right) \rightarrow(R, r)$ for all object $(R, r)$ in $R(X)$ constitutes a natural transformation $[m]: \tilde{F} \tilde{G} \rightarrow 1_{R[X] \text {. }}$

THEOREM 4.4. If every monomorphism in $\mathscr{M}^{*}$ is a split monomorphisms $[8 ; p .19]$, then $R\{Y\}$ is equivalent to $R[X]$, i.e., $[m]: \tilde{F} \tilde{G} \rightarrow 1_{R[X]}$ is a natural isomorphism.

The functor $\pi_{A}: \mathcal{C}^{A} \rightarrow \mathcal{C}$ in Example 2.8 has a left adjoint $\Delta_{A}: \mathcal{C} \rightarrow \mathcal{C}^{A}$ since $\mathcal{C}$ has products. In the case $\mathcal{C}=$ Set $_{0}$, the category of nonempty sets, we have $\operatorname{Set}_{0}\left[\pi_{A}\right] \cong$ $\operatorname{Set}_{0}\left\{U_{A}\right\}$ (an equivalence of categories) because every monomorphism in $\mathscr{H}^{*}=\langle\mathbf{M o n}\rangle_{a \in A}$ is a split monomorphism. This case was initially observed by Kato [6, Theorem 5.1].

\section{References}

[1] Arbib, M. A. and Manes, E.G.: Arrows, structures, and functors, The categorical imperative, Academic Press, New York, 1975.

[2] - Foundations of system theory: The Hankel matrix, J. Comput. System Sci., 20 (1980), 330-378.

[3] $:$ Generalized Hankel matrices and system realization, SIAM J. Math. Anal., 11 (1980), 405-424.

[4] Ehrig, H., Kreowski, H.-J., Maggiolo-Schettini, A., Rosen, B. K. and Winkowski, J. : Transformations of structures: an algebraic approach, Math. Systems Theory, 14 (1981), 305-334.

[5] Huzino, S.: Foundations of mathematical programmings (Japanese), Asakura Shoten, Tokyo, 1979.

[6] KATO, A.: A categorical approach to the representative independency of relational data models and their application to dependencies (Japanese), Kyoto Univ. Sûrikaisekikenkyûsho Kôkyûroku No. 423 (1981), 275-293.

[7] - An abstract relational model and natural join functors, Bull. Infromatics and Cybernetics, 20 (1983), 95-106.

[8] Mac Lane, S.: Categories for the working mathematicans, Springer-Verlag, New York, 1971.

[9] TAKeshima, T.: On a generalization of relational data model dependencies by first order predicates satisfying equivalence laws (Japanese), Kyoto Univ. Sûrikaisekikenkyûsho Kôkyûroku No. 423 (1981), 257-274.

Communicated by S. Arikawa

Received October 12, 1983 